\title{
ZNF432 is over-expressed in brain metastatic breast cancer.
}

Shahan Mamoor, $\mathrm{MS}^{1}$

1shahanmamoor@gmail.com

East Islip, NY USA

One study reported the incidence of central nervous system metastases in breast cancer patients treated with trastuzumab as $34 \%{ }^{1}$. We mined published microarray data ${ }^{2,3}$ to discover genes associated with brain metastasis in breast cancer. We identified significant differential expression of ZNF432 in patients with breast cancer. ZNF432 may be relevant to the biology underlying colonization of the brain with metastatic breast cancer clones.

Keyword: breast cancer, metastasis, brain metastasis, central nervous system metastasis, ZNF432, systems biology of breast cancer, targeted therapeutics in breast cancer. 
One study reported a 34\% incidence of central nervous system metastases in patients with breast cancer treated with the monoclonal antibody trastuzumab (Herceptin) ${ }^{1}$. Treatment options for patients with brain metastatic breast cancer are limited ${ }^{4}$. We mined published microarray datasets ${ }^{2,3}$ to understand in an unbiased and systems-level fashion genes whose expression most significantly distinguished brain metastases from primary tumors of the breast, the cells from which these metastatic clones are presumably derived. We found significant differential expression of ZNF432 in the brain metastases of patients with brain metastatic breast cancer.

\section{Methods}

We utilized microarray datasets GSE $125989^{2}$ and GSE100534³ for this differential gene expression analysis of brain metastatic breast cancer in conjunction with GEO2R. GSE100534 was generated using Affymetrix Human Gene 1.0 ST Array technology with $n=16$ primary tumors from patients with breast cancer and $n=3$ brain metastases from patients with brain metastatic breast cancer. GSE43837 was generated using Affymetrix Human X3P Array technology with $n=19$ primary breast tumors and $n=19$ brain metastases from patients with breast cancer, analysis performed using platform GPL1352. The Benjamini and Hochberg method of $p$-value adjustment was used for ranking of differential expression but raw $p$-values were used to assess statistical significance of global differential expression. Log-transformation of data was auto-detected, and the NCBI generated category of platform annotation was used. A statistical test was performed to evaluate whether ZNF432 gene expression was significantly between primary breast tumors and brain metastases in humans with breast cancer using a two-tailed, unpaired t-test with Welch's correction. We used PRISM for all statistical analyses of differential gene expression in human breast cancer (Version 8.4.0)(455).

\section{Results}

We mined published microarray data ${ }^{2,3}$ to discover genes associated with brain metastasis in breast cancer.

\section{ZNF432 is differentially expressed in brain metastatic breast cancer.}

We identified ZNF432 as among the genes whose expression changes most significantly when comparing brain metastases to primary tumors of the breast in patients with brain metastatic breast cancer $^{2}$ (Table 1). When sorting each of the genes expressed in brain metastases based on significance of change as compared to primary tumors of the breast, ZNF432 ranked 592 out of 22277 total transcripts (Table 1), equating to $97.3 \%$ differential expression. Differential expression of ZNF432 in brain metastases in brain metastatic breast cancer was statistically significant (Table $1 ; p=1.05 \mathrm{E}-02$ ).

We queried a second microarray dataset ${ }^{3}$ to validate transcriptome-wide differential expression of ZNF432 in brain metastatic breast cancer. We again found that ZNF432 was among the genes whose expression changed most significantly when comparing metastases of the brain to primary tumors of the breast in patients with breast cancer (Table 2). When sorting each of the genes expressed in brain metastases based on significance of change as compared to primary tumors of the breast, ZNF432 ranked 5883 out of 33297 total transcripts (Table 2), equating to $82.3 \%$ differential expression. Differential expression of ZNF432 in brain metastases in brain metastatic breast cancer was statistically significant (Table $2 ; p=2.43 \mathrm{E}-02$ ). 
ZNF432 is expressed at significantly higher levels in brain metastases as compared to primary tumors of the breast in brain metastatic breast cancer.

We obtained exact mRNA expression values for ZNF432 from brain metastases and primary tumors of the breast to evaluate magnitude and direction of change in ZNF432 expression in brain metastases in patients with breast cancer. ZNF432 was expressed at higher levels in brain metastases in patients with breast cancer and this difference was statistically significant (Figure $1 ; p=0.0013$ ). We calculated a mean fold change of $1.17 \pm 0.12$ in ZNF432 expression when comparing brain metastatic tissues to primary tumors of the breast (Table 1).

Thus, we found that ZNF432 was among the genes, globally, whose expression was most different when comparing primary tumors of the breast to brain metastases in patients with breast cancer.

\section{Discussion}

There is limited published information describing functions for ZNF432. In a study of the adenosine deaminase acting on RNA, ADAR1, depletion of ADAR1 resulted in up-regulation of ZNF4325. A single-nucleotide polymorphism in ZNF432 was associated with bronchodilator response to albuterol in children with asthma, and inhaled corticosteroids influenced this association, modulating degree of bronchodilator response based on ZNF432 genotype 6 .

We reported here that the gene encoding ZNF432, a zinc finger protein of undescribed function, was among the genes whose expression was most quantitatively different in metastases to the brain when compared to primary tumors of the breast in patients with metastatic breast cancer. ZNF432 may be of relevance to the biology underlying any number of steps important for metastasis to the brain: exit from the breast, traversing the vasculature and the blood brain barrier, and colonization of the brain. 


\section{References}

1. Bendell, J.C., Domchek, S.M., Burstein, H.J., Harris, L., Younger, J., Kuter, I., Bunnell, C., Rue, M., Gelman, R. and Winer, E., 2003. Central nervous system metastases in women who receive trastuzumab-based therapy for metastatic breast carcinoma. Cancer, 97(12), pp.2972-2977.

2. Iwamoto, T., Niikura, N., Ogiya, R., Yasojima, H., Watanabe, K.I., Kanbayashi, C., Tsuneizumi, M., Matsui, A., Fujisawa, T., Iwasa, T. and Shien, T., 2019. Distinct gene expression profiles between primary breast cancers and brain metastases from pair-matched samples. Scientific reports, 9(1), pp. $1-8$.

3. Schulten, H.J., Bangash, M., Karim, S., Dallol, A., Hussein, D., Merdad, A., Al-Thoubaity, F.K., AlMaghrabi, J., Jamal, A., Al-Ghamdi, F. and Choudhry, H., 2017. Comprehensive molecular biomarker identification in breast cancer brain metastases. Journal of translational medicine, 15(1), p.269.

4. Robson, M., Im, S.A., Senkus, E., Xu, B., Domchek, S.M., Masuda, N., Delaloge, S., Li, W., Tung, N., Armstrong, A. and Wu, W., 2017. Olaparib for metastatic breast cancer in patients with a germline BRCA mutation. New England Journal of Medicine, 377(6), pp.523-533.

5. Wang, I.X., So, E., Devlin, J.L., Zhao, Y., Wu, M. and Cheung, V.G., 2013. ADAR regulates RNA editing, transcript stability, and gene expression. Cell reports, 5(3), pp.849-860.

6. Wu, A.C., Himes, B.E., Lasky-Su, J., Litonjua, A., Peters, S.P., Lima, J., Kubo, M., Tamari, M., Nakamura, Y., Qiu, W. and Weiss, S.T., 2014. Inhaled corticosteroid treatment modulates ZNF432 gene variant's effect on bronchodilator response in asthmatics. Journal of Allergy and Clinical Immunology, 133(3), pp.723-728.

PAGE 4 
PAGE 5 
PAGE 6 


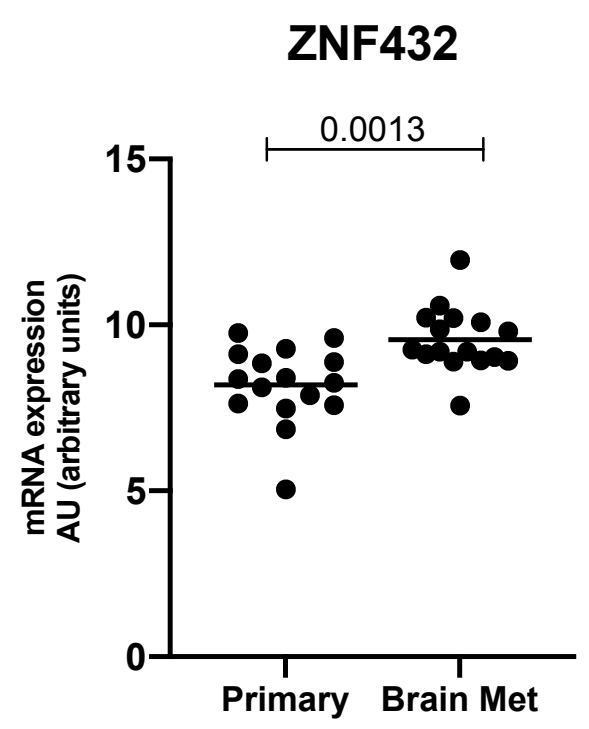

Figure 1: ZNF432 is expressed at significantly higher levels in brain metastases as compared to primary tumors of the breast in patients with brain metastatic breast cancer.

The mRNA expression level of ZNF432 in primary tumors of the breast (left) and in the brain metastases of brain metastatic breast cancer patients (right) is graphically represented here with mean ZNF432 levels marked and the result of a statistical test evaluating difference in mRNA expression level between brain metastases and primary tumors of the breast, a $p$-value, listed above. 